\title{
Self Disclosure, Autonomy and Connection: An Analysis of Texting In Romantic College Relationships
}

\author{
Kim Flottemesch, Kristi Loobeek, Jennifer Schultz, and Elliot Powell \\ Concordia University- St. Paul
}

\begin{abstract}
College students in the United States are quickly becoming the experts of technological advancements and their implementation into everyday life. Arguably one of the most popular advancements within the last decade is the use of Short Message Service (or texting) with mobile phones. The researchers chose to investigate how texting, within a romantic couple of the college generation, affected the relationship. Specifically, the researchers explored the effect texting had upon self-disclosure and the balance between autonomy and connectedness within a couple. Participants of the study hailed from a faith-based institution of higher education in an urban area of the Midwestern United States. The study thoroughly addressed the relationship between the frequency of texting and its aftereffects on both self-disclosure and the dyadic tension between autonomy and connection.
\end{abstract}

\section{INTRODUCTION}

The use of mobile phones is a common topic of research and analysis, and yet there is much to be learned. As the technology of the $21^{\text {st }}$ century evolves, so does the means of communication. With omnipresent social media driving society into new communication patterns, the uses and style of usage concerning mobile phones is rapidly changing. Leading the changes of technology and communication are the younger populations of the world. Looked to as emerging experts in the field of social media and technology, college students possess a second-nature understanding of all things technological and are therefore a prime age group to investigate.

This study looked to shine a light on the trends of the college students while using mobile phones with a romantic partner and its aftereffects. Texting (the sending of a short electronic message from one phone to another, generally 140 characters or less) is one of the most recent communication trends. This study is designed to answer the following questions: 1.)Will frequent texting positively benefit the growth of the relationship, or does it create a disconnect?; 2) Do partners feel they can self-disclose through texting?; and 3)Can texting jump the gap of media richness and become beneficial to the process of self-disclosure in relationships?

The study explores the relationship between the frequency of texting as related to couple connectedness versus autonomy and frequency as it relates to the rate of self-disclosure. Through the introduction of mobile phones to a relationship, the rate of self-disclosure can be accelerated to a previously unattainable level compared to face-to-face communication. Likewise, the balance of autonomy and connectedness within a romantic couple is potentially hindered by the overuse/underuse of mobile phones. The lack of message richness in texting and its effect on relationship closeness is also examined. To address these aspects, the researchers chose two theories to help explain the data: Social Information Processing Theory and the Social Penetration Theory.

After reviewing related research on the topic, the researchers hypothesized that an increased frequency of texting between romantic partners would result in an increased level of self-disclosure. In addition, the researchers hypothesized that, with an increased frequency, the texting would stimulate connectedness to a state only achieved through computer-based communication called hyperpersonal perspective.

The researchers saw this study as valuable to the academic community for multiple reasons. Initially, the researchers found importance in the study due to the limited research previously conducted on mobile phones as they relate to romantic relationships. Secondly, the researchers felt the need to investigate the influences the new age of technology, specifically mobile phones, has on the relational dialectic of autonomy versus connection in romantic relationships. Finally, the researchers believed the data collected would be able to aid the investigation into perpetual contact studies.

\section{LITERATURE REVIEW}

\section{Introduction}


Looking into mobile phone communication and romantic relationships encompasses many other subsections of investigation. Mobile phones themselves are complicated pieces of technology that allow for more means of communication than strictly voice telecommunication. One of the newer areas of communication with technology is Short Message Service (SMS), or texting as it will be referred to from now on, which allows for a message of generally less than 140 characters to be sent from one mobile device to another. Combining this use of technology with relationships proves to have multiple areas of discussion. There are many different facets of a romantic relationship, but to narrow it down, this study focuses on how frequency of texting affects the dynamic of autonomy versus connection and self-disclosure. To further narrow the study, only college students were surveyed.

\section{Mobile Phones}

"The use of cell phones to communicate is quickly becoming one of the most common and easiest ways to connect with others" (Coyne et al., p.2). The reasons of preference for mobile phone use varies. The youngadult generation is an emerging expert on the different means of communication involving mobile phones (Coyne et al., 2011). A study conducted involving this generation found they preferred mobile phone conversations and text messaging over face-to-face communication because it gave them the option of: talking to multiple people at once, leaving large gaps in the conversation, concealing truth, and being able to immediately clarify misunderstandings (Madell\&Muncer, 2007). They claimed the misunderstandings stemmed from the lack of media richness when using mobile phones. On the positive side, they felt mobile communication gave them more time to "ponder and articulate what they were trying to express" (Coyne et. al., p.2).

Specifically concerning text messaging, the lack of vocal tone, facial cues, and other nonverbal communication indicators were found to be the main cause of the aforementioned "misunderstandings" (Madell\&Muncer, 2007). Even so, because more communication between partners tends to indicate better relational functioning, greater use of mobile phones is beneficial to relational partners, despite the negativity of the topics discussed (Jin \& Pena, 2010). Furthermore, "recent evidence indicates that people can strengthen their family bonds, facilitate friendships, and build mutual support via mobile communication" (Jin \& Pena, p.40).

\section{Emerging Adult Relationships and Perpetual Contact}

The emerging adult group is classified as "individuals between the ages of 18 and 29, who are neither adolescents nor adults, but who are in the process of becoming an adult" (Demir, p.2). As explained by Demir (2007), the formation and maintenance of romantic relationships are central in the lives of emerging adults. While most of these individuals are enrolled in a college at that point in time, the university environment provides a wealth of opportunities to socialize and develop relationships. A study found that most college students consider their romantic involvements as the closest relationships they have (Demir, 2007). Differences in romantic relationships in emerging adults versus adolescents are that adolescents generally pursue romantic relationships for "fun and experiencing companionship and do not last very long" (Demir, p.2); whereas relationships in emerging adults are "serious, more intimate and committed" (Demir, p.2).

Within the realm of college students, the ways in which free time is spent ranked "sleeping" and "faceto-face communication" one and two respectively, while "texting" came in third (Hanson et al., 2011). The high ranking of this mobile phone usage can be acquainted to the phrase "perpetual contact," coined by Katz and Aashus (2009). In a society driven by technology, it has become an accepted norm that people will be at our constant communication beck and call. "By removing the requirement of being in a fixed location, cell phones give freedom to relational partners to contact one another at any time" (Duran, p.21). With communication between partners only a click away, it raised the question of when there is time for "appropriate contact" (Katz \&Aashus, 2009). "Thus, the perpetual contact capability of cell phones potentially strains relationships, creating too much connection at the expense of autonomy" (Duran, p.21). With so much potential for self-disclosure, a romantic couple might find the pressure of constant communication intimidating and struggle with the rate at which they should self-disclose information.

Individuals in romantic relationships were found to use mobile phones more frequently than those not in romantic relationships (Jin \& Pena, 2010). Trending with both face-to-face communication and mobile communication is that more frequent mobile or face-to-face communication was significantly associated with less perceived loneliness. With this increased use of mobile phones, normally consisting of short and frequent calls and text messages, feelings of connectedness between communicators were reaffirmed.

\section{Autonomy Versus Connection}

Within the dialectical perspectives study developed by interpersonal communication scholars, Baxter and Montgomery, four strategies were established in order to manage the dialectical tensions experienced within relationships. The four basic strategies included: 
(a) selection, in which partners choose to make one of the contradictory poles the dominant one, such as emphasizing autonomy over connection; (b) separation, which occurs when partners try to keep the opposing forces separate such as by emphasizing one pole for some topics[ ...] (c) neutralization, which is a strategy that involves trying to reduce the intensity of the contradictory pulls as in trying to choose a moderate or middle position; and (d) reframing, in which relational partners attempt to redefine a dialectical tension to diminish the sense of opposition. (Duran, Kelly, Rotaru 2011)

Rather than thinking of the dialectical tensions as a negative, Baxter and Montgomery encouraged others to simply see them as "inherent in social life" (p.7). These four factors are constantly at play when analyzing the romantic relationships; however, the imbalance and fluctuation of autonomy versus connection is especially prevalent.

The way the couple chooses to balance these dialectical tensions is reflected in their choice of either: selection, separation, neutralization or reframing. Each strategy poses positives and negatives to its outcome, such as using selection and emphasizing connection over autonomy when using mobile phones may cause an abrupt loss of autonomy and individual identity (Duran et al., 2011). Jonathan Pettigrew(2009) also identified the tension of autonomy versus connection, (often referred to as independence vs. dependence) within the use of mobile phones in close interpersonal dyads. Individuals were able to "use text messaging to both assert autonomy and to maintain connectedness with their relational partners" (p.697). However, studies have found there are strong differences in the text messaging patterns, content, and mutual understanding when looking at non-romantic dyads versus romantic pairs; the text messages are interpreted in different ways when sent to different partners (Pettigrew, 2009).

The contradictory and simultaneous needs of partners to feel connected to one another and to remain independent individuals is what characterizes romantic relationships (Duran, Kelly, Teodora, 2011). To be in a successful relationship each person needs to be able to "forfeit some degree of autonomy; at the very least they must consider the needs and impact of their own actions on their relational partners" (p.21). At the same time, if all autonomy is lost the relationship will be destroyed due to the loss of identity of each individual partner. Rawlins in 1992 described this paradox of the need for independence and dependence as the freedom to be independent and act without the relational partner's interference, and the freedom to be dependent- that is, to be able to rely on or call on the partner for assistance or involvement in one's life.

\section{Self DisclosureThrough Mobile Phones}

Within the scope of romantic relationships, the most common reason for couples to text message one another is to "discuss serious issues, approach a potentially confrontational subject, express affection, or to hurt their partner" (Coyne, Stockdale, Busby, Iverson, Grant, 2011). However, the element of self-disclosure via texting is an interesting sub topic. Within relationships, the trading of personal information through selfdisclosure is a fundamental building block. The rate with which information is disclosed may vary from couple to couple; however, the use of mobile phones and text messaging is becoming a prominent channel of this type of communication.

Strong relationships rely on both partners participating in frequent communication. Through the use of mobile phones, and the theory of perpetual contact, partners can accomplish their needs to communicate through text messaging or voice telecommunication. Regular cell phone usage and relationship satisfaction have been linked and found to be strongly and positively correlated (Miller-Ott\& Duran, 2012). The more frequently romantic dyads engaged in communicating through mobile phones, the higher satisfaction levels each partner experienced. "Greater use of mobile voice calls with a romantic partner was associated with lower relational uncertainty and more love and commitment" (Jin \& Pena, 2010)

While the main purpose of mobile phone usage is to communicate, the reduction of uncertainty is also a focal point during the initial interaction between romantic couples (Emmers\& Canary, 1996). The struggle to reduce uncertainty through communication, and yet assert autonomy from the relational partner, is resolved by the creation of rules to cell phone usage developed within romantic pairs. A study looking at these mobile phone rules by Jin and Pena in 2010, found that couples who had set rules of appropriate contact were positively correlated with relationship satisfaction. The couples who laid out the appropriate calling and texting times along with times when it was unacceptable to not answer an incoming call were more secure in their relationship together (Jin \& Pena, 2010). These rules then play a role on the self-disclosure process. Self-disclosure becomes only appropriate at certain times. The study conducted by Jin and Pena also found that couples were more likely to start a potentially confrontational conversation through text message rather than face-to-face or voice telecommunication.

\section{Conclusion}

To conclude, the use of mobile technology has opened new areas of study when it comes to romantic relationships. The rate of self-disclosure can be altered by the theory of perpetual contact and potentially harm a 
relationship. The study looked into the frequency of texting as it relates to both self-disclosure rates as well as the relational dialectic between autonomy and connectedness. The researchers concluded that a more frequent use of texting led to a higher rate of self-disclosure. They also concluded that frequent texting in romantic couples created more connection than autonomy. Through this research, couples can also learn how to use mobile technology to connect in a way that strengthens their relationship.

\section{RESEARCH QUESTIONS}

When looking at individuals in relationships, the use of text messaging can either be a positive daily staple or a means of staying relatively unattached from the significant other. This study primarily focused on the use of mobile phones in self-disclosure within romantic couples: the rate at which they disclose information and the frequency of daily texting.

RQ1: How does texting influence self-disclosure within romantic relationships of college students?

The researchers conducted this study in an effort to break ground on a previously unexplored aspect of mobile phones and their effect of romantic dyads. Drouin and Landgraff's study in 2012 supported the idea that texting a romantic partner has an element of distanced emotional quality. This theory was also supported by Pettigrew (2009) stating thattexting can help both "assert autonomy and promote closeness" (Pettigrew, p.2). In this case, texting can be a hindering element to a romantic relationship. This study will investigate the relational dialectic between autonomy versus closeness.

RQ2: Does texting on a regular basis within these relationships stimulate interconnectedness or autonomy?

Current research fails to focus on college students and their texting habits within romantic relationships. This study surveyed emerging adults to decipher if they feel texting significant others helps cement their relationship or creates a distancing insecurity.

\section{METHODOLOGY}

The researchers, in an effort to distribute and collect as many surveys as possible, decided to use the online survey-generator website named Survey Monkey. Through the use of the website, the researchers were able to input the survey questions into an electronic format. The final version of the survey was assigned a web address by Survey Monkey. This address was then disseminated to the student body through the University's email system.

\section{Participants}

The participants of the survey were required to be undergraduate students of the selected university. Of the 1,291 surveys distributed, 292 were completed. This gave the researchers a $22.6 \%$ response rate. Of the respondents, 145 were female and 68 were male. The majority of students indicated they were seniors in college with $27.1 \%$. However, of the 292,79 were disqualified from consideration due to one or more of the following factors: 1) did not own a mobile phone $(\mathrm{N}=3)$; 2) had not been in a romantic relationship during their college time $(\mathrm{N}=67)$; 3) were currently married $(\mathrm{N}=17)$. Multiple students were disqualified by more than one restriction.

\section{Procedures}

The researchers constructed the survey through the website Survey Monkey. The survey was given a web address to direct students to the questions. After the survey went live and was emailed to students, it remained open for 36 days. The survey was self-administered by the students and contained no extra incentive. Within the email was a short paragraph explaining the purpose of the study and the length of time the survey would take. Below the paragraph was a hyperlink that lead to the survey.

The survey consisted of 28 questions total. The first five questions inquired about the participants' gender, year in school, possession of a cell phone, relationship history in college, and marital status through a multiple choice format. Respectively, the latter three questions also supplied an "other" option with an open response box. Each of the remaining 23 questions contained a Likert scale and had the following options: no/never, sometimes, often, very often, yes/always. A setting within Survey Monkey required that only one answer be selected per question and no question/s could be skipped.

The survey questions focused on the thoughts, feelings and actions of the participant, rather than the participant's significant other. The results were analyzed with three main components in mind: self-disclosure, autonomy versus connection, and frequency. Participants were asked if they would send/receive at least 25 texts with their significant other a day. The results from this question were then cross tabulated with a number of different questions to achieve chi-square test results comparing frequency to either self-disclosure or autonomy/connection. 


\section{RESULTS}

The entire undergraduate student body of the university was surveyed asking 28 questions to determine if a correlation existed between the amount of texting students do with their significant others and the amount of self-disclosure experienced in the relationship. The researchers also wanted to uncover if texting on a regular basis would stimulate connectedness or autonomy. Of the 1,291 undergraduate students sent the survey at the university in the fall of 2012, 354 participated in this study by responding to the survey (a response rate of 29\%). Of those 354, 62 participants did not complete the entire survey, leaving 292 remaining participants. 87 participants also did not qualify for the research because they had not been in a romantic relationship during college $(\mathrm{N}=67)$, were married at the time of the study $(\mathrm{N}=17)$, did not own a cell phone $(\mathrm{N}=3)$, or some combination of the above. This left 213 students (67\%) attending the university to remain active participants of this study. The results of the qualified participants were then analyzed.

To test the first hypothesis, the researchers compared participants' ratings of the number of texts they share with their significant other to how likely they are to self-disclose with that person. A chi-square contingency (or chi square test with association) indicated the two significantly correlated $(\mathrm{P}<.01)$. The researchers reject the null hypothesis and accept the alternative.

To determine if participants felt an obligation to self-disclose, they were asked if they felt pressured to share personal information since their partner had done so. A majority of participants $(52.1 \%)$ responded "No/Never". When comparing this rating with the rating of number of text messages sent to a significant other, a chi square test with association indicated no evidence of significant association or relationship between these variables $(\mathrm{P}>.05)$. These findings failed to reject the null hypothesis.

Participants were also asked if they would avoid using text messaging to discuss some topics because they would rather talk about them in person. A majority of participants (35.7\%) responded "Sometimes". When comparing this rating with the rating of a number of text messages sent to a significant other, a chi square test with association indicated no evidence of significant association or relationship between these variables $(\mathrm{P}>$.05). These findings failed to reject the null hypothesis.

A final question to determine if participants felt an obligation to self-disclose asked participants if they would ever choose to text about an issue instead of talking in person because they felt less pressure through text messaging. A majority of participants (42.3\%) responded "Sometimes" with "No/Never" just behind (38.5\%). A chi square test with association indicated no significant relationship among these variables $(\mathrm{P}<.05)$. These findings also fail to reject the null hypothesis.

To test the second hypothesis, researchers compared participants' ratings of the number of frequent texts they share with their significant other to how secure or stable they felt within their relationship. A majority of participants (39.4\%) responded "Sometimes". When comparing this rating with the rating of number of text messages sent to a significant other, a chi square test with association indicated a significant relationship between these variables $(\mathrm{P}<.05)$. These findings support the hypothesis and reject the null.

The next question regarding autonomy and connectedness also supported the hypothesis. Participants were asked if texting with their significant other helped them feel connected although they were apart. Participants mostly answered "Often" (24.4\%), "Very Often" (25.4\%), or "Yes/Always" (27.7\%). A chi square contingency (or chi square test with association) indicated the two significantly correlated $(\mathrm{P}<.01)$.

A chi square contingency also indicated significant correlation $(\mathrm{P}<.01)$ when participants were asked if frequent texting with their significant other made them feel as though they were "on the same page". A majority (36.6\%) responded "Sometimes", supporting the hypothesis and rejecting the null.

Participants were also asked if they felt pressured to text their significant other throughout the day. A majority of participants (51.0\%) responded "No/Never". When comparing this rating with the number of text messages sent, a chi square contingency indicated a significant association between these variables $(\mathrm{P}<.05)$. The data seems to reject the null hypothesis.

The last question pertaining to the relationship between the frequency of text messaging and autonomy/connection asked participants if they viewed texting as a means of being controlled by their significant other. A majority of participants $(83.6 \%)$ responded "No/Never". A chi square test with association indicated no evidence of a relationship between these variables $(\mathrm{P}>.05)$. These findings failed to reject the null hypothesis.

\section{DISCUSSION}

Social Information Processing Theory

Social Information Processing theory (SIP) investigates how relationships can be established through the use of technological advances (Walther, 1992). A key component of SIP is computer-mediated communication (CMC) or text based communication. Such messages can be shared through email, letters, texting, instant messaging and multiple other media. Due to the fact that these messages are strictly text, 
messages become stripped of non-verbal cues. It is this absence of cues, and, yet, the development of relationships through a piece of technology that attracted the researchers. The question of how people are able to build romantic relationships through such a simple and unforgiving channel requires attention and thought.

Of the qualified participants, $94.2 \%$ replied they would text with their significant other throughout the day. The researchers found only $19.9 \%$ of respondents said they never sent/received at least 25 text messages to their significant other throughout one day. The majority of the participants replied "Sometimes" with $31.2 \%$. In second place was "Yes/Always" with 22.8\%. The researchers used the response to this second question ("Would you send/receive at least 25 texts with your significant other a day?") as a building block for their more extensive analysis. The question served as a basis to build the term "frequent" upon. By positively answering the question that they sent/received at least 25 text messages per day, the researchers then cross-tabulated the question to analyze the effects this "frequent" texting had upon different aspects of relationships, specifically: self-disclosure and the balance between autonomy and connectedness.

Relationships, in general, need a multitude of communication to grow and evolve. Different types of communication can facilitate this growth easier than others. Face-to-face interaction is the richest form of communication (Daft, Lengel, and Trevino, 1987). Whereas, text only messages, with long response rates, is the leanest form of communication. The richness of the message is classified by the amount of non-verbal cues that can be transferred through the channel. A study in 2007 suggested misunderstandings while texting directly stemmed from this lack of richness (Madell\&Muncer). This scale of categorizing communication channels is key when comparing and describing the growth of relationships through CMC. The data collected supported that, although the channel of communication was exceptionally lean, the participants did not fail to self-disclose to their significant others. Of the qualified participants, 36.7\% ( $\mathrm{N}=114)$ replied they were always comfortable telling personal information about themselves to their significant other through texting. The question was also cross-tabulated against the aforementioned frequency question in order to form a correlation between the two (frequency and self-disclosure). The results of the chi-square test supported H:1 in hypothesizing more frequent texting would increase the amount of self-disclosure. The p-value for the two questions was (.00). With a significant correlation number being (.05) or below, this value reaffirmed $\mathrm{H}: 1$.

One of Walther's key points of SIP was that through the exchange of interpersonal information (selfdisclosure), an impression of the other person would be formed--equivalent to those formed for face-to-face communication (Walther, 1992). It is through this impression formation, whether text-based or face-to-face, that we develop relationships. The study conducted looked into if the exchange of interpersonal information could be done through a cell phone and yet yield the same results as face-to-face disclosure could. Participants were also asked if they felt comfortable sharing personal information about themselves while texting with their significant other. With a p-value of .00009 , our results showed a strong correlation between how often participants text their significant other and how comfortable they felt sharing personal information. Participants who said they always texted their romantic partner 25 times a day or more were more apt to feel comfortable telling personal information about themselves. This can be explained by the hyperpersonal perspective of SIP. Because CMC allows romantic partners to grow closer, they feel more comfortable sharing information. This allows a comfort level, a trust factor where this personal information is safe. The data supported that participants who responded they text their significant other very often also reported a strong sense of comfort in sharing personal information. Whereas participants who reported they sometimes or never texted their partner 25 times a day showed great discomfort sharing personal information (see figure 1.2).

Aiding in the development of a hyperpersonal perspective is the perpetual contact theory developed by Duran in 2011. Because cell phones allow for contact and communication at any given time and location, the amount of self-disclosure is far greater than simply face-to-face communication. Duran suggested couples would struggle with the amount of appropriate self-disclosure; however, the data from the recent study suggested otherwise. A large number of participants show they are comfortable discussing almost anything over text-based communication. With the rapid growth in technology, current college students have grown up using computers and cell phones and feel fluent in their usage. The students are extremely knowledgeable about phone use and are at ease using them as a form of communication. When participants were asked if they would avoid texting about some topics, preferring to discuss them face to face, there was little correlation between the two--indicating once again, that participants felt comfortable self-disclosing via text. However, the data also supported that non-frequent texters also felt equally as comfortable self-disclosing when they did indeed text. While frequency of texting with romantic partners may not affect whether they are more comfortable discussing some topics in person, the study supported they are still comfortable using text-based communication to discuss things with their partner. Duran also concluded perpetual contact would strain relationships and "[create] too much connection at the expense of autonomy" (p.21). However, in the recent study, the researchers found $41.8 \%$ $(\mathrm{N}=89)$ of participants responded they would never feel anxious if they didn't text their significant other throughout the day. This led the researchers to believe that even though participants had achieved the hyperpersonal perspective, they also had managed to maintain some individual autonomy. The study suggested 
the nature of the topic may have a stronger impact on whether they prefer to discuss face-to-face or over text messaging than the frequency of their text-based communication.

When participants were asked if they ever chose to text about an issue because they felt less pressured through the use of mobile phones, frequent texters did not show a strong inclination to do so. Conversely, non-frequent texters showed a stronger correlation to text over face-to-face communication for possibly controversial topics. The researchers concluded the non-frequent texters, who do not use text messaging as a main source of communication, may not feel as though text-based communication is a personal as face-toface communication. As a result, they may utilize text messaging to discuss difficult issues due to its impersonal manner. Students who text frequently and have built a bond with their significant other through text-based communication do not feel text messaging relieves any pressure because, to them, it is a standard form of communication. The findings of the recent study added to the research done by Jin and Pena in 2010. While Jin and Pena found couples were more likely to start a potentially confrontational conversation through text messaging, this study found this was only true with non-frequent texting romantic couples.

In his hyperpersonal perspective, Walther (1996) explains how the sender, receiver, and channel have a significant impact on the intimacy level of relationships developed through text based communication. Because the two people in the relationship are not face to face, the sender is able to filter out attributes and share their more positive thoughts and qualities. The receiver is also more likely to seek out similarities between themselves and the sender, dismissing less favorable traits. This allows members of a relationship to feel they are on the same page with each other and create a stronger sense of intimacy. This phenomenon is explained in SIP through the manipulation of the sender/receiver channel relationship. Walther explained the sender would enable "selective self-presentation," in other words, they would only share positive qualities about themselves. Due to this overly positive assimilation of characteristics, the couple will develop social identitydeindividuation (SIDE) (Spears and Lea, 1992). In SIDE, the two people in the relationship will cease having individual identities and become a merged couple. Duran, Kelly and Teodora recognized the SIDE process in their 2011 study and concluded the contradictory and simultaneous needs of partners to feel connected to one another and yet remain independent individuals is what will cause the relationship to fail if the scale tips too far towards the side of connection. Participants who texted more than 25 times a day showed responded they felt as though they were on the same page more often than those who texted less frequently.

The cross-tabulation of frequency and the question concerning connection ("Did texting with your significant other help you feel connected even when you were apart?"), found there was a strong correlation between the two. Participants who reported they always or very often texted at least 25 times a day were more likely to respond they always or very often felt connected to their significant other when they were apart. Participants who sometimes or never texted 25 times a day with their partner showed texting was less likely to help them feel they were connected when they were apart. Because text messaging is an asynchronous channel of communication, time becomes a significant factor. If a student sends a text message to their romantic partner and that person responds immediately or shortly after, they may continue to feel connected in spite of distance. Walther (1992) also notes asynchronous interaction allows the receiver time to plan, contemplate and edit their comments before responding to the initial message. This time to ponder and contemplate was also found as a main beneficiary factory by Madell and Muncer in their 2007 study. This again allows the sender to present themselves in a more positive light, in turn creating a stronger perceived connection.

\section{Social Penetration Theory}

Self-disclosure is accomplished most effectively when given slowly and in a reciprocated manner. With the exchanging of personal information, one can begin to feel more connected to another person. This is the basic premise of the Social Penetration Theory developed by Irwin Altman and Dalmas Taylor (1987). The theory can be visually represented by the image of an onion. At the core of the onion is one's true self-concept. As the layers of the onion approach the surface they become more superficial and public. When relating this model of the onion to a relationship, one may think of the outer layers as the small talk one does when first getting to know someone. However, when engaging in a romantic relationship, the wedge of conversation driven into the onion, also known as the penetration of self-disclosure, goes deeper into the onion than most other relationships (Taylor and Altman, 1987). Topics of conversation may move to more serious issues such as "goal, aspirations, religious convictions, or deeply held fears and fantasies". Participants replied these topics found in the outer layer were the topics of most text conversations with their significant other. The majority of the participants $(29.6 \%)$ responded that they often texted about light topics such as "how your day is going, what you are doing late, likes and dislikes..."

Because social penetration is more rapid at the beginning and slows with time, people may feel more pressure to share something about themselves at the beginning of a relationship. At this time, the information being shared is surface level. Participants who reported texting their significant other more than 25 times a day did not feel pressured to share information about themselves if their partner had shared something with them. 
The researchers concluded couples who text frequently are more likely to be at a stage in their relationship where they are comfortable with each other and have shared many of their core values. They are secure in their relationship and do not feel the need to share something every time their partner does. However, students who text their significant other less frequently may not have built up that level of connection yet. They reported feeling more pressure to share information but it is likely this may be more surface level information. They are in the getting-to-know each other stage of their relationship and are still peeling back the layers of each's personality structure.

The strongest correlation was in the connection of how often participants text messaged their significant other and how secure and stable they felt within their relationship. This correlation would suggest that participants who are texting frequently have built a deep connection and are secure in their relationship. They use text messaging as a form of self-disclosure, continually reciprocating information. These romantic couples have gone through many layers of social penetration. While they are texting often, they are sharing day-to-day information, slowly letting the other person into their inner core. Because there is a constant flow of communication via text messaging, the results suggest they feel a sense of connectedness to their significant other. Participants who frequently texted their romantic partner reported feeling less pressure to text their significant other during the day. The study suggested that these students already felt connected to their partner and were secure in their relationship. Although they had established a strong connection, the frequent texters did not have trouble being autonomous. This strategy of balancing dialectical perspectives was explained in Baxter and Montgomery's study: rather than thinking of the dialectical tensions as a negative, they encourages others to simply see them as "inherent in social life" (p.7). The strategy selected to deal with these tensions was also reflected in the recent study's findings. While the researchers found the participants did indeed lean more towards the selection of connection over autonomy, not all individual identity was lost. $49.8 \%(\mathrm{~N}=106)$ of participants said they did not feel pressured to text their significant other throughout the day, suggesting that, although perpetual contact has made it possible for couples to text at all times, participants felt able to go without that kind of connection. This finding was supported by the previous research done by Pettigrew in 2009. His conclusion matched the researchers': individuals were able to "use text messaging to both assert autonomy and to maintain connectedness with their relational partners" (p.697).

\section{LIMITATIONS}

A limitation this study encountered was the unaddressed use of instant messaging on cell phones. Through applications like Facebook, smartphone users are capable of sending text only messages that are more commonly classified by young-adults as "messaging" rather than "texting." Other technology that includes messaging is Skype. However, Skype can combine a text element with the ability to see another through a live video feed. This multifaceted technological innovation makes incorporating data into the study difficult. Due to the survey size and localized area of participants, the study is not comparable nationwide. Participants that indicated themselves as "married," have not engaged in a romantic relationship, or do not own a cell phone also limited the amount of results available.

\section{SUGGESTIONS FOR FUTURE RESEARCH}

The results of the survey have provided a building block for more extensive research. Any retrospective studies done in the future should be conducted in a different subsection of the continental United States in order to provide a more accurate representation of college students. While the researchers were able to identify trends in correlation with frequency of texting, these may be offset by results from a different area.

Future research could also explore the use of Skype and other video calling programs within romantic relationships. Within the Social Information Processing Theory, computer mediated communication would now include this advancement in technology. How the introduction of a video feed affects the interactions and frequency of communication should be researched.

Lastly, communication through mobile phone entertainment applications should be investigated. Applications such as Draw Something, Words with Friends and Snapchat are one of the fastest growing means of interaction through technology. This new interaction, paired with texting, voice telecommunication or video feedscouldyieldsignificant findingstotheacademiccommunity. 


\section{REFERENCES}

[1] Coyne, S. M.; Stockdale, L.; Busby, D.; Iverson, B.; and Grant, D. M. (2011) "I luv u :)!” A Descriptive Study of the Media Use of Individuals in Romantic Relationships. Family Relations, 2, 150-162

[2] Daft, R., Lengel, R., and Trevino, L. K. (1987). Message Equivocality, Media Selection, and Manager Performance: Implications for Information Systems. MIS Quarterly, 11, 355-365

[3] Drouin, M. andLandgraff, C. (2012). Texting, Sexting, and Attachment in College Students' Romantic Relationships. Computers in Human Behavior, 28, 444

[4] Duran, R. L.; Kelly, L.; and Rotaru, T. (2011). Mobile Phones in Romantic Relationships and the Dialectic of Autonomy Versus Connection. Communication Quarterly, 59, 19-36

[5] Hanson, T.L.; Drumheller, K.; Mallard, J.; McKee, C.; and Schlegel, P. (2011). Cell Phones, Text Messaging, and Facebook: Competing Time Demands of Today's College Students.College Teaching, 59, 23-30

[6] Hertlein, K.M. and Webster, M. (2008). Technology, Relationships, and Problems: A Research Synthesis. Journal of Marital and FamilyTherapy, 34, 445-460

[7] Jin, B. and Pena, J F. (2010). Mobile Communication in Romantic Relationships: Mobile Phone Use, Relational Uncertainty, Love, Commitment, and Attachment Styles. Communication Reports, 23, 39-51

[8] Ling, R. and Baron, N.S. (2007). Text Messaging and IM: Linguistic Comparison of American College Data. Journal of Language and Social Psychology, 26, 291

[9] Madell, D.E., \&Muncer, J.J. (2007). Control over social interactions: An important reason for young people's use of the Internet and mobile phones for communoication? CyberPsychology and Behavior, 10, $137-140$

[10] Miller-Ott, A. E.; Kelly, L.; Duran, R. L. (2012) The Effects of Cell Phone Usage Rules on Satisfaction in Romantic Relationships. Communication Quarterly, 60, 17

[11] Pettigrew, J. (2009). Text Messaging and Connectedness Within Close Interpersonal Relationships. Marriage \& Family Review, 45, 697

[12] Spears, R. and Lea, M. (1992). Social Influences and the Influence of the Social. In Lea, M. (ed.), Contexts of Computer-Mediated Communication (30-65)

[13] Taylor, T. and Altman, I. (1987). Communication in Interpersonal Relationships: Social Penetration Processes. In Roloff, M. and Miller, G. (Eds.), Interpersonal Processes: New Directions in Communication Research (259). Newbury Park, CA: Sage

[14] Walther, J. (1992). Interpersonal Effects in Computer Mediated Interaction: A Relational Perspective. Communication Research, 19, 52-90 